\title{
Positional uncertainty in lateral masking and the perceptual superiority of words
}

\author{
LEO D. GEOFFRION \\ The Johns Hopkins University, Baltimore, Maryland 21218
}

\begin{abstract}
The possible role of positional uncertainty as a basis for lateral masking and the perceptual superiority of words was examined. The stimuli were five-letter strings, of which the middle three letters were the targets and the end letters were distracting flankers which were positioned either adjacent to the target or separated from it. The trigram targets were of three types (words, pseudowords, and nonwords). The positional uncertainty of individual letters was varied through the use of two response modes, with 18 college students participating in each mode. One group used a response mode which did not allow transpositions of letter sequence, while the other group had no such restriction. The results showed that positional uncertainty affected the magnitude of lateral masking but not that of the word superiority effect, suggesting that different processes underlie these two phenomena. Error analyses within response mode as well as response bias comparisons further confirmed this conclusion.
\end{abstract}

When asked to identify a complex visual display, a person must resolve two major forms of uncertainty before an accurate identification is possible. These are discriminative and positional uncertainty. In other words, a person must decide not only what features were present in the stimulus but also the spatial arrangement among these features. An error in either or both of these factors will result in an incorrect identification.

Most research on visual information processing has focused on discriminative uncertainty and has assumed, at least implicitly, that any stimulus changes which affect target identification do so by changing the discriminative uncertainty of the target. This assumption neglects the possibility that accuracy shifts may also result from changes in the uncertainty about how elements are arranged in the display.

This experiment attempts to examine the possible role of positional uncertainty in two perceptual phenomena. The first is lateral masking, which is the decrease in target identification accuracy resulting from embedding the target among distractors. The second is the perceptual superiority of words wherein letters within words are easier to identify than the same letters embedded within nonwords.

Most explanations of lateral masking have assumed that the flanking distractors make the target harder to identify through an increase in discriminative uncertainty. Shaw (1969), for example, proposed a model where the flanking letters reduce the processing time available to the target, thereby reducing the number of features that can be successfully identified.

\footnotetext{
The author acknowledges the guidance and assistance of Drs. Howard Egeth and James Pomerantz. This work was supported by an N.I.M.H. predoctoral research fellowship and by a contract from the Office of Naval Research. Please send reprint requests to: Leo Geoffrion, Department of Education, Morrill Hall, University of New Hampshire, Durham, New Hampshire 03824.
}

Others have suggested that lateral masking is based on a process analogous to lateral inhibition commonly found in neural studies of visual receptors (Bouma, 1970; Walley \& Weiden, 1973; Wolford \& Hollingsworth, 1974a). This approach claims that the flanking letters directly inhibit the detection of visual features in a manner similar to the way stimulation of one retinal detector inhibits stimulation of adjacent detectors.

More recently, Eriksen and his colleagues (Eriksen \& Eriksen, 1974, Eriksen \& Hoffman, 1973) have claimed that lateral masking reduces accuracy, not by making the target harder to identify, but by increasing the uncertainty about which letter within the display constitutes the target (i.e., increased positional uncertainty). This was demonstrated by contrasting the masking effects of three different types of flanking letters in a two-button discrimination task. Flanking the target with letters of the same response set had no measurable effect, while flanking the target with letters of the opposite response set greatly increased response latency. The use of flankers from neither response set produced intermediate results. These results were obtained even when the subject knew in advance where the target would appear in the stimulus. They concluded that lateral masking is the result of positional uncertainty producing competition among incompatible responses.

While this experiment is very interesting and informative, it leaves considerable doubt as to whether the main locus of lateral masking lies in positional uncertainty or in the response competition that it evokes. If positional uncertainty is the principal cause of lateral masking, then a clearer demonstration of its effects is desirable.

In similar fashion, early models of the perceptual superiority of words emphasized the belief that words 
were easier to identify because of reduced discriminative uncertainty. This has usually taken the form of some type of "unitization hypothesis," wherein the individual letters of a word combine into some form of higher order unit that is more recognizable than the individual letters.

It is possible, however, that the basis for the word superiority effect lies not in a reduction of discriminative uncertainty, but rather in a reduction of positional uncertainty. Estes $(1975 a, b)$ has proposed two mechanisms by which positional uncertainty could cause a word superiority effect. The first possibility is that people possess a response bias favoring letter combinations which conform to normal spelling patterns. This bias is present even when subjects have no advance expectation that the stimulus will form a word.

Estes. Allmeyer, and Reder (1976) demonstrated this response bias by examining confusions in letter sequence among four-letter consonant strings. They found that highly directional digraphs such as " $\mathrm{CH}$ " or " $\mathrm{CK}$ " were more accurately reported when presented in their more usual order (i.e., "CH") than in their reversed order ("HC"). This finding is similar to earlier work by Frith (1971), who found that as children learn to read they do not become more adept at recognizing a letter in its correct orientation, but rather they develop a response bias toward the correct letter orientation.

Estes (1975b) suggested that when a subject expects the stimulus to be a word, this bias favoring words should be even greater because subjects are then able to use their knowledge of spelling patterns to reduce their uncertainty about letter sequences. Estes compared subjects' accuracy in discriminating between the letters " $L$ " and " $R$ " when embedded within either words or nonwords. In half of all trials, neither letter appeared in the cued position. If one examined the percentage of correct identifications of the cued letter, the usual word superiority effect was found in that the word context produced greater accuracy than did the nonword context. On the other hand. if one examined only those trials where the subject gave either an " $L$ " or " $R$ " as a response, then no difference in accuracy was found between the two contexts $(97 \%$ vs. $95 \%)$. Error analysis revealed that the word superiority effect resulted only from the decreased incidence of transposition errors in the word context condition. He therefore concluded that the word superiority effect was based not on improved discriminability of the letters in words but because of reduced positional uncertainty about the order of the letters in words arising from a person's knowledge of spelling patterns in the language.

Estes finding of no difference in discriminability between letters in the word and nonword contexts would have been more convincing had the letters " $L$ " and " $R$ " not been so dissimilar. The incidence of confusions between them was always less than $5 \%$ for all conditions, which is so low that floor effects may have masked any differences between words and nonwords.

A second problem with this approach is that error analysis provides, at best, only an indirect proof of this hypothesis. A more direct test would be to manipulate positional uncertainty experimentally. If it is the basis for the word superiority effect, then in conditions where positional uncertainty is minimized, the word superiority effect should be greatly diminished.

The goal of this experiment was to directly test the role of positional uncertainty in these two phenomena. These two were chosen because Estes (Estes, Allmeyer, \& Reder, 1976; Note 1) has recently sought to explain both lateral masking and the word superiority effect through a common process of letter identification centering on positional uncertainty. It is therefore valuable to examine whether both lateral masking and the word superiority effect share the same reliance upon positional uncertainty.

To test this hypothesis, two groups of subjects used different response modes for the identical stimulus strings. One group (fixed sequence response mode) was made explicitly aware of serial position constraints used to construct the stimuli. This was done both by direct instructions as well as by the use of a response device which permitted discriminative errors but did not allow any inversions of letter sequence. The other group (variable sequence response mode) was given no such instructions and used a response system which allowed them to make both types of errors. If positional uncertainty is the basis for these two phenomena, then subjects in this second group should show much greater effects in both phenomena since they possess greater uncertainty about the letter sequence of the stimuli.

\section{METHOD}

\section{Subjects}

Eighteen subjects participated in each of the two response modes. with no subject participating in both modes. All were paid volunteers from a university population. Most were experienced observers. having participated in ether experiments, but all were naive about the purposes and goals of this one. All reported having normal or fully corrected vision in both eyes.

\section{Stimuli}

The stimuli were five-letter strings. They were centered at $2.5 \mathrm{deg}$ to the right or left of a central fixation dot because previous research has shown that this angle produces the largest word-nonword difference (Bouma, 1973). All letters were made from 14-pt Futura Demi-Bold dry transfer lettering (Prestype No. 1282). At a viewing distance of $30 \mathrm{~cm}$, each letter subtended a vertical angle of $0.60 \mathrm{deg}$ with a stroke width of $0.13 \mathrm{deg}$.

The middle three letters of each string constituted the target and were positioned with a 0.13 -deg gap between adjacent contours. The first and fifth letters of the stimulus constituted the flankers. In half of all stimuli, the flankers were separated from the target letters by $0.13 \mathrm{deg}$ between adjacent contours (narron' spacing). while in the other hall the separation was $0.70 \mathrm{deg}$ (wide spacing).

The trigram targets were made from a set of 12 letters. such that 
4 letters could appear in each of the three serial positions. The first letter was always A, D, 1 , or T; the second letter was always $C, O$. $R$. or $U$ : and the third letter was always $E, G, K$. or $Y$. This produced 63 target strings falling into three target types. Sixteen were common w'ords such as "DOG" or "TOY." Twenty constituted orthographically normal but meaningless strings (pseudowords) such as "ACY" or "DRE." Finally, 27 were neither meaningful nor orthographically normal (nonwords) such as "ACG" or "IOY." One string "AOK" was not used since, although orthographically anomalous, it has become a meaningful idiom in some areas. The ratings of orthographic normality were validated using an independent sample of 14 raters (Geoffrion. 1975).

This target structure offers an additional advantage in that each letter is about equiprobable for each target type. This avoids the possibility that any differences among target types could be attributed to differences in the detectability of individual letters (cf. Smith \& Jones. 1970).

The flanking letters were randomly drawn from the remaining letters of the alphabet except for the letters " $Q$ " and " $Z$." The two flanking letters were always different from each other and the selection of flankers was matched for the two spacing conditions.

A total of 126 stimuli were generated with all 63 target strings appearing in both spacing conditions.

\section{Procedure}

All subjects were tested individually and were told that this was an experiment to determine how well they could read strings of letters presented to the left or right of their visual fields. Special emphasis was taken to avoid the terms "word" or "spelling patterns" in order to reduce their expectancy for viewing words (cf. Aderman \& Smith, 1971). The stimuli were presented using a two-channel tachistoscope.

Eighteen subjects in the fixed sequence response (FSR) mode were taught how to indicate their responses using a response board which did not permit sequence inversions. The board consisted of a wooden frame within which three wooden slats could slide to reveal the various letter combinations through three horizontally arranged windows. On each slat were printed only those letters which could actually appear in that serial position. Particular emphasis was taken to point out that different letters appeared in each serial position and that all targets could be constructed from combinations available on the response board.

The second group of 18 subjects was the variable sequence response (VSR) mode. They indicated their responses using a set of 12 metal chips about $2.5 \mathrm{~cm}$ square, on each of which was mounted one of the 12 possible target letters. They were told that all possible target combinations could be constructed from these 12 chips but were given no information about the serial position constraints used in target construction. After each trial, the experimenter rearranged the chips to prevent accidental discovery of the target structure.

Each subject received 15 practice trials, during which accuracy feedback was given. They then viewed the main stimuli in four blocks of about 32 trials each without feedback. Between each block, exposure was adjusted to maintain accuracy at about a $50 \%$ of trials totally correct level (chance level was less than $2 \%$ for both groups). Exposure was never more than $100 \mathrm{msec}$ in order to prevent the subject from moving his eyes and foveally viewing the stimulus. All experimental conditions (two spacings by three target types by two visual fields of presentation) were randomly mixed using a fixed order of presentation for all subjects.

\section{RESULTS}

For the purpose of data analysis, each serial position was treated independently. The main dependent variable was the percentage of trials where the letter in a particular serial position was correctly identified. These percentages were arcsine transformed and analyzed using a mixed-design analysis of variance with one between-subjects variable (response mode) and three within-subjects variables (spacing, target type, and serial position). No attempt was made to analyze effects separately in each visual field.

The grand mean was $75 \%$ correct letter identification in each serial position. Since chance is $25 \%$ for the FSR mode (chance for the VSR mode is somewhat less), the observed results should be free of range limitation problems. The following terms were found to be significant at or beyond the .05 êrror level.

Response mode: $\mathrm{F}(1,34)=8.31, \mathrm{p}<.01$. Subjects in the FSR mode were more accurate overall than those in the VSR mode in spite of efforts by the experimenter to maintain constant accuracy between groups by varying exposure duration. The average exposure for the FSR mode was $60 \mathrm{msec}$, while in the VSR mode it was 90 msec.

Spacing: $\mathrm{F}(1,34)=180.00, \mathrm{p}<.001$. Subjects were considerably more accurate when the flanking letters were spaced farther away from the target $(65.5 \%$ vs. $84.4 \%$ for the narrow and wide spacing conditions). This confirms the existing lateral masking literature in that flanking the target with distracting letters markedly reduces accuracy.

Response Mode by Spacing: $\mathrm{F}(1,34)=19.98$, $p<.001$. Scheffé post hoc tests revealed that in the wide spacing condition there was no difference between groups, while in the narrow spacing condition accuracy decreased for both groups but the decrease was greater for the VSR mode than for the FSR mode $(26.1 \%$ vs. $11.7 \%)$. The implications of this interaction will be presented in the discussion section.

Target type: $\mathrm{F}(2,68)=9.61, \mathrm{p}<.001$. Scheffé tests showed that there was no difference between words and pseudowords $(76.0 \%$ vs. $75.9 \%)$, but that both were more accurate than nonwords $(73.0 \%)$. This main effect is not in itself very interesting since it can be attributed to any of a variety of causes, including response bias, short-term memory coding differences, and the perceptual superiority of words. The better indicator of perceptual superiority would be interactions between target type and spacing.

Target Type by Spacing: $\mathrm{F}(2,68)=7.22, \mathrm{p}<.005$. Scheffé tests showed that in the narrow spacing condition there was no difference among the three target types, whereas in the wide spacing condition words and pseudowords were significantly more accurate than nonwords (Table 1). The implications of this interaction will be presented in the discussion section.

Response Mode by Serial Position: $\mathrm{F}(2,68)=8.30$. $p<.001$. Examination of the means for each serial position revealed that in the FSR mode subjects were equally accurate for all serial positions while in the 
Table 1

Percentage Correct for Each Target Type and Spacing

\begin{tabular}{lcc} 
& \multicolumn{2}{c}{ Spacing } \\
\cline { 2 - 3 } Target Type & Narrow & Wide \\
\hline Words & 65.5 & 86.6 \\
Pseudowords & 66.2 & 85.6 \\
Nonwords & 64.9 & $81.0^{*}$ \\
\hline
\end{tabular}

* Significantly less than the other two target types, $p<.05$ (Scheffé post-hoc test). The minimum difference necessary for significance is $4.2 \%$.

VSR mode accuracy decreased systematically from left to right. Analysis of variance conducted separately on each group confirmed this hypothesis, $F(2,34)=$ 2.88. n.S., vs. 8.26, p <.005, respectively, for FSR and VSR modes. This interaction can be attributed to possible differences between groups in response latency or response order as well as differential effects of positional uncertainty on different serial positions. Unfortunately, the experiment does not permit one to discriminate among these possibilities.

Spacing by Serial Position: $F(2,68)=6.17$, $\mathrm{p}<.005$. The wide spacing increased accuracy for all serial positions, but this increase was greater for the end letters than for the middle letter $(15.5 \%$ vs. $11.6 \%)$. This difference is consistent with previous research in lateral masking which found that the facilitative effect of inserting spaces affected primarily the immediately adjacent letters (Estes \& Wolford, 1971; Shaw, 1969; Wolford \& Hollingsworth, 1974b).

The interaction of Response Mode by Target Type was not significant $[F(2,68)<1]$. Likewise no higher order interactions involving these two variables proved significant. Thus, differences in response mode do not appear to result in substantial differences in the magnitude of the word superiority effect.

Since the lack of a significant interaction between response mode and the word superiority effect suggests that positional uncertainty is not a major factor in the word superiority effect, it is important to examine the two processes by which positional uncertainty is assumed to produce this effect.

The first of these processes was the belief that subjects have reduced positional uncertainty for orthographically normal strings. To examine this, a separate analysis was performed on those errors that were sequence errors in the VSR mode. Only the VSR mode was used since sequence errors were not possible in the FSR mode. A sequence inversion was defined as a response in any one serial position that corresponded to a letter which had actually appeared in a different location. Thus, for example, if the stimulus were "TUE," then the response "TOU" would be scored as one sequence inversion in the third serial position, while the response "UTE" would be scored as one sequence inversion in the first serial position and one in the second.
The incidence of sequence inversions was also divided into the first and second halves of the experiment to test whether subjects in the VSR mode learned the stimulus constraints during the experiment.

Sequence errors did decrease from the first to the second halves of the experiment $(12.3 \%$ vs. $10.9 \%)$. but the decrease was not statistically significant $[F(1,17)=1.84$, n.s. $]$. Thus it is unlikely that subjects in the VSR mode substantially learned the stimulus constraints. In addition, all subjects in the VSR mode were asked at the end of the experiment whether they thought that certain letters had always appeared in the same serial positions. Most reported notiving only one or two such letters.

Other signiticant terms in the analysis of variance for sequence inversions revealed the following: sequence errors were less likely in the wide spacing condition $[F(1.17)=177.03, \mathrm{p}<.001]$. They were also more likely for the middle letter than for the end letters $[F(2.34)=37.13, p<.001]$.

There was no significant main effect for target type. nor were any higher order interactions of target type significant, even though a substantial word superiority effect was found for this group $[F(2.34)=$ $5.43, \mathrm{p}<.011$. Thus Estes' claim that the word superiority effect is based on reduced positional uncertainty for words was not supported by this experiment.

The second major aspect of Estes' model was that subjects possess a response bias favoring orthographically normal letter sequences and that when unsure of a letter sequence they will transpose it into an orthographically normal sequence. If this were true, then one would expect that subjects would make more word responses than expected from the stimulus frequency and that this bias would be greater in those conditions with greater positional uncertainty. To test this, all responses by both groups were classified into three categories: words, pseudowords, and nonwords. Analysis of variance of the data summarized in Table 2 showed there was a significant shift in responses between the narrow and wide spacing conditions $[F(2.68)=17.76, p<.001]$. Scheffé post hoc tests showed that, in the narrow spacing condition, subjects made fewer word responses and more nonword responses than expected from the stimulus distribution, suggesting a response bias against words. In the wide spacing condition, subjects made fewer nonword responses than expected, while the number of word and pseudoword responses did not differ from the stimulus distribution, suggesting only a minimal bias favoring orthographically normal sequences. Thus it is possible that the results noted earlier, wherein the word superiority effect was obtained only for the wide spacing condition, may be the result of a shift in response bias between the two spacing conditions. However, if one chooses to adopt 
Table 2

Frequency of Stimuli and Responses for Each Target Type and Spacing

\begin{tabular}{lccc} 
& \multicolumn{3}{c}{ Target Type } \\
\cline { 2 - 4 } & Word & Pseudoword & Nonword \\
Stimulus & & Narrow Spacing \\
Response & 15.9 & 19.9 & 26.7 \\
Bias $\dagger \dagger$ & 13.2 & 19.8 & 29.5 \\
& $-2.7^{*}$ & -.1 & $2.8^{*}$ \\
Stimulus & & Wide Spacing & \\
Response & 15.9 & 19.9 & 26.9 \\
Bias $\dagger \dagger$ & 16.5 & 20.8 & 25.4 \\
\hline
\end{tabular}

tStimulus frequency is noninteger because of occasional spoiled trials. Maximum possible frequency is 16,20 , and 27. respectively.

† Bias equals response frequency minus stimulus frequency:

* Minimum difference for significance $(p<.05)$ is 1.2 using Scheffé post-hoc test.

this position, it becomes necessary to accept a hypothesis considerably weaker than that of Estes, since in neither condition did subjects show a clear response bias favoring words and pseudowords. Furthermore. a response bias favoring nonwords was found in the narrow spacing condition, which is the condition where subjects had greater positional uncertainty, as shown by the increase in sequence inversions for this condition. This is the opposite to what the Estes model had predicted.

\section{DISCUSSION}

The key to understanding the results of this experiment is the interaction of target type and spacing (Table 1). This interaction suggests a two-stage processing model for target recognition. The first stage involves the isolation of the unit for analysis, while the second stage involves detailed processing to identify the structure of the units isolated by the first stage (cf. Neisser, 1967, preattentive vs. focal attentive processes).

Lateral masking primarily disrupts the first stage in that surrounding the target by flanking elements prevents the first stage from rapidly isolating the target. The interaction of response mode by spacing demonstrates that positional uncertainty plays a major role in lateral masking. This interaction showed that the facilitative effect of inserting spaces around the target was much greater for those subjects with greater positional uncertainty (VSR mode). This difference between groups can be completely accounted for by the decrease in sequence errors between the narrow and wide spacing conditions for the VSR mode. Since identical stimuli were presented to both groups, it is doubtful that the observed interaction can be plausibly accounted for by a differential shift in discriminative uncertainty.

Although the experiment demonstrates the importance of positional uncertainty in lateral masking, it does not eliminate the possibility that the insertion of spaces also produces a decrease in discriminative uncertainty, since ven subjects in the FSR mode showed a significant space facilitation effect. Lateral masking may involve changes in both discriminative and positional uncertainty.

In the second stage of processing, the units isolated by the first stage are analyzed in detail. It is in this second stage that knowledge of spelling patterns becomes a significant aid to target recognition. Word superiority effects were not found in the narrow spacing condition (Table 1) because the first stage was unable to isolate the trigram target from its tlankers. If the second stage were required to process the five-letter string as a single unit, then all the strings would be treated as nonwords, since few, if any, of them formed orthographically normal units.

The effect of positional uncertainty appears to be localized to the first stage, since it had no measurable effect on the magnitude of the word superiority effect. Not only was the direct role of positional uncertainty in the word superiority effect not confirmed, but the two processes by which positional uncertainty was presumed to produce the word superiority effect were not confirmed. Subjects in this experiment did not show a reduced positional uncertainty for words, nor did they demonstrate a response bias in their favor.

It may be possible that the role of positional uncertainty becomes greater when comparing targets longer than three letters. Trigrams, particularly in the wide spacing condition, may be unrepresentative in that two of the three letters appear "anchored" in that they appear adjacent to a space. Perhaps in longer words uncertainty about the arrangement of the middle letters leads to an increased role for positional uncertainty in the word superiority effect. Some support for this position comes from the work of Eichelman (1970), who found that the magnitude of the word-nonword difference increased with increasing word length. Nevertheless this experiment demonstrates that positional uncertainty cannot be the only factor underlying the word superiority effect, since the effect is observable even in situations where positional uncertainty is greatly reduced.

Finally, this experiment suggests that recent attempts to explain both lateral masking and the word superiority effect through a common process centering on positional uncertainty will not be fruitful since the two processes exhibit different relationships to positional uncertainty. A two-stage model as suggested above provides a more parsimoneous explanation for the differing characteristics of lateral masking and the word superiority effect. 


\section{REFERENCE NOTE}

1. Estes. W. Colloquium presentation at the Johns Hopkins University. February 1975.

\section{REFERENCES}

Aderman. D., \& Smith, E. Expectancy as a determinant of functional units in perceptual recognition. Cognitive Psychology. 1971, 2. 117.129.

BoumA, H. Interaction effects in parafoveal letter recognition. Nature, 1970, 226, 177-178.

BoumA, H. Visual interference in the parafoveal recognition of initial and final letters of words. Vision Research, 1973, 13, 767-782.

Eichelman, W. Familiarity effects in the simultaneous matching task. Journal of Experimental Psychology, 1970, 86, 275-282.

ERIKSEN, B., \& ErIKSEN, C. Effects of noise letters upon the identification of a target letter in a nonsearch task. Perception \& Psychophysics, 1974, 16, 143-149.

Eriksen, C., \& Hoffman, J. The extent of processing noise elements during selective encoding from visual displays. Perception \& Psychophysics, 1973, 14, 155-160.

Estes. W. On the locus of inferential and perceptual processes in letter identification. Journal of Experimental Psychology: General, 1975, 104, 122-145. (a)

Estes. W. Memory, perception and decision in letter identification. (Paper presented at the Third Loyola Symposium on Cognitive Psychology, May 1974.) In R. Solso (Ed.), Information processing and cognition: The Loyola Symposium. Potomac, Md: Erlbaum Associates. 1975. (b)
Estes. W., Allmeyer, D., \& Reder, S. Serial position functions for letter identification at brief and extended exposure durations. Perception \& Psychophysics, 1976, 19, 1-15.

Estes, W., \& Wolford, G. Effects of spaces on report from tachistoscopically presented letter strings. Psychonomic Science, 1971. 25. 77.80.

Frith, U. Why do children reverse letters. British Joumal of Psychology, 1971, 62, 459-468.

Geoffrion, L. Lateral masking and the word superiority effect. J.S.A.S. Catalog of Selected Documents in Psychology, 1975, 5, 321.

NeIsser, U. Cognitive psychology. New York: Appleton-CenturyCrofts, 1967.

SHAw, P. Processing of tachistoscopic displays with controlled order of characters and spaces. Perception \& Psychophysics. $1969,6,257-266$.

Smith, C., \& Jones, D. Sequential dependencies in letter search. Journal of Experimental Psychology, 1970, 85, 56-60.

W ALLEY. R., \& WEIDEN, T. Lateral inhibition and cognitive masking: A neuropsychological theory of attention. Psychological Revien'. 1973, 80, 284-302.

WOLFORD, G., \& HollingsworTh, S. Lateral masking in visual information processing. Perception \& Psychophysics, 1974, 16. 315-320. (a)

Wolford, G., \& Hollingsworth S. Retinal location and string position as important variables in visual information processing. Perception \& Psychophysics, 1974, 16, 437.442. (b)

(Received for publication November 24. 1975; revision received January $15,1976$. 